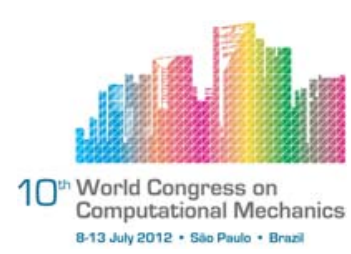

\title{
Nonlinear harmonic analysis of structures containing joints with impacts and friction
}

\author{
D. Schurzig ${ }^{1}$, L. Panning-von Scheidt ${ }^{1}$, A. Hartung ${ }^{2}$, J. Wallaschek ${ }^{1}$ \\ ${ }^{1}$ Institute of Dynamics and Vibration Research, Leibniz Universität Hannover (schurzig@ids.uni- \\ hannover.de)
}

${ }^{2}$ MTU Aero Engines GmbH, Munich

\begin{abstract}
An algorithm is proposed which can be applied to finite element models in order to efficiently perform nonlinear harmonic analyses without neglecting nonlinear effects due to joint friction and impacts. In order to decrease the size of the nonlinear differential equation to be solved, the proposed algorithm reduces the structural matrices of the system to be investigated using the Craig-Bampton method after importing them from commercially available FE codes. Depending on the geometrical setup and materials of the system joints, different discretization assumptions can be applied. The implemented contact model is based on Coulomb friction and Newton impact constraints which are being determined by a linear complementarity problem (LCP) formulation. The computation itself is performed in time domain using a modified Newmark time stepping approach for numerical stability even if the time step size chosen is fairly large. Both the mathematical approach itself as well as numerical results are presented for the simple system of a cylindrical beam in contact with a rigid wall in order to give an in-depth description of the proposed algorithm.
\end{abstract}

Keywords: Nonlinear harmonic analysis, joints, impacts, friction, linear complementarity problem

\section{INTRODUCTION}

Finding the eigenfrequencies and corresponding mode shapes of systems undergoing harmonic excitation is highly important for increasing the systems durability. In order to do so, system developers generally use FE software - which is a quick and generally reliable way to determine a system's characteristics. Components can be imported from CAD software or created directly with the FE software, several kinds of joints can be applied to certain features of the structure to be analyzed, and finally the structure's dynamic behavior can be determined, e.g. by performing a harmonic analysis. However, the application of joints to a structure generally means that the degrees of freedom (DOF) of the surfaces or bodies in contact are limited in a way that mirrors the behavior of an ideal joint, that is without considering impacts or friction. Thus, energy dissipation due to joint friction as well as certain kinds of component wear cannot be analyzed since the causes of these phenomena do no longer exist 
within the simulation model. Most importantly, if designing a system or trying to improve its behavior, joint components and surfaces should be chosen in a way that most efficiently decreases vibrational amplitudes in order to extend the system's lifetime as much as possible. Therefore, a computation method is necessary that does not simulate joints as limited DOF, but fully recognizes the nonlinear interaction of the involved body features as impacts with friction. Since doing so entails a significant increase in computational effort, other assumptions, simplifications and discretizations should be applied in order to keep computation times at a moderate level.

The algorithm proposed in this paper receives the structural information of the component or system of interest from a commercial FE code. Thus, it should be seen as an extension of the abilities of FE software rather than an entirely new program code. In a first step the structural matrices, i.e. the nonlinear differential equations to be solved, are being modally reduced using the Craig-Bampton method [1] in order to increase computational efficiency. This particular type of modal reduction technique allows to physically preserve certain master DOF while modally describing all other DOF whose physical information is not being needed during computation. The proposed algorithm then solves the forced response problem without neglecting nonlinear effects by utilizing a contact model depicting impacts as well as stick and slip friction. Both impact and friction models are implemented in an LCP formulation. In addition, different rigid body assumptions for contact zone discretizations can be applied to the interacting structures. Thus, the number of master DOF necessary to accurately describe the components being in contact can be minimized to further decrease computation time. Since all computations are being executed in time domain, the Newmark time stepping scheme [2], which is popular among FE programmers due to its numerical stability, has been modified in order to accurately follow both the Newton impact and Coulomb friction models. Since the behavior of the system to be analyzed is nonlinear, subharmonic responses have to be expected and recognized. In order to do so, periodic system responses are being determined utilizing a modified Poincare mapping approach. Thus, the proposed algorithm represents an efficient and important FE code extension for nonlinear harmonic analysis of both single components and systems including joints with clearance.

\section{SYSTEM DESCRIPTION AND SIMPLIFICATION}

To depict the functionality of the proposed algorithm in a vivid manner, every part of the algorithm is being described on a representative model of a revolute joint which is modeled as a cylindric beam inside a bushing (see Fig. 1 A). This setup shows the same geometric features while cutting out possibly complex system properties which could distract from the actual joint simulation task. For a further breakdown of the problem, the bushing has additionally been substituted by a rigid wall - as shown in Fig. 1 B - in order to bring the basic problem to the foreground: Modeling states of separation, impacts, stick and slip friction.

In our case, the structural matrices are being obtained from the FE software ANSYS, but could also be generated using other available FE codes. This step should be followed by a reduction of the structural matrices. In order to not lose all physical information about the system by performing a regular modal reduction of the structural matrices, the Craig-Bampton reduction method [1] was implemented which allows to preserve predefined master DOF as physical 

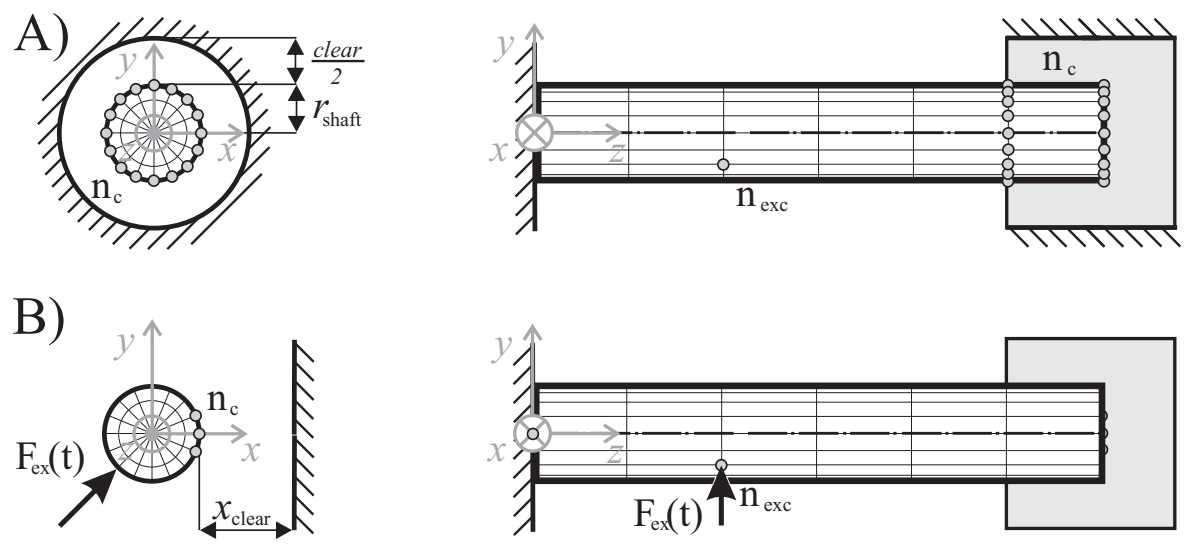

Figure 1. Simplified versions of a revolute joint showing a beam A) inside a cylindrical bushing and $\mathrm{B}$ ) next to a rigid contact wall

information within the reduced structural matrices while modally describing all other DOF. For the system shown in Fig. 1, the master DOF are the ones belonging to the nodes labeled as $n_{c}$. While all nodal information along the two highlighted edges of the beam would be necessary in case of the contact partner being the bushing (A), only the few nodes facing the rigid wall have to be considered for case B. Fig. 1 shows three contact nodes per edge for case $\mathrm{B}$ to also recognize a possible torsional deformation of the beam. Thus, if looking at a nonlinear differential equation of motion of kind

$$
\mathbf{M} \ddot{\mathbf{q}}(t)+\mathbf{D} \dot{\mathbf{q}}(t)+\mathbf{K q}(t)=\mathbf{F}_{\mathrm{ex}}(t)+\mathbf{R}(t)
$$

with mass, damping and stiffness matrices $\mathbf{M}, \mathbf{D}$ and $\mathbf{K}$, harmonic excitation force $\mathbf{F}_{\text {ex }}(t)$ and contact force $\mathbf{R}(t)$, the vector containing the system's DOF $\mathbf{q}(t)$ can be written as

$$
\mathbf{q}(t)=\left[\begin{array}{l}
\mathbf{q}_{\mathrm{m}}(t) \\
\mathbf{q}_{\mathbf{s}}(t)
\end{array}\right]
$$

where the indices $m$ and s stand for master and slave DOF respectively. In order to built up the transformation matrix according to [1], the constraint modes $\Psi$ and component modes $\boldsymbol{\Phi}$ have to be determined using

$$
\left[\begin{array}{cc}
\mathbf{K}_{\mathrm{mm}} & \mathbf{K}_{\mathrm{ms}} \\
\mathbf{K}_{\mathrm{ms}}^{\mathrm{T}} & \mathbf{K}_{\mathrm{ss}}
\end{array}\right]\left[\begin{array}{l}
\mathbf{E} \\
\boldsymbol{\Psi}
\end{array}\right]=\left[\begin{array}{c}
\mathbf{Q} \\
\mathbf{0}
\end{array}\right] \text { with } \boldsymbol{\Psi}=-\mathbf{K}_{\mathrm{ss}}^{-1} \mathbf{K}_{\mathrm{ms}}^{\mathrm{T}}
$$

and

$$
\left(\mathbf{K}_{\mathrm{ss}}-\omega_{i}^{2} \mathbf{M}_{\mathrm{ss}}\right) \boldsymbol{\phi}_{i}=\mathbf{0} \text { with } \boldsymbol{\Phi}=\left[\boldsymbol{\phi}_{1} \boldsymbol{\phi}_{2} \ldots \boldsymbol{\phi}_{p}\right] .
$$

Herein, the vector $\Phi$ controls the magnitude of the reduced order structural matrices depending on how many component modes $\phi_{i}$ are included. The resulting transformation matrix

$$
\mathbf{T}=\left[\begin{array}{ll}
\mathbf{E} & 0 \\
\boldsymbol{\Psi} & \boldsymbol{\Phi}
\end{array}\right]
$$

can then be applied to Eqn. (1) by multiplication according to

$$
\begin{aligned}
\tilde{\Theta} & =\mathbf{T}^{\mathrm{T}} \boldsymbol{\Theta} \mathbf{T} \\
\tilde{\boldsymbol{\Gamma}} & =\mathbf{T}^{\mathrm{T}} \boldsymbol{\Gamma}
\end{aligned}
$$


in which $\Theta$ and $\Gamma$ stand for the structural matrices $\mathbf{M}, \mathbf{D}$ and $\mathbf{K}$ and the excitation and contact force $\mathbf{F}_{\mathrm{ex}}(t)$ and $\mathbf{R}(t)$ respectively. The reduced order equation of motion can then be stated as

$$
\tilde{\mathbf{M}} \ddot{\tilde{\mathbf{q}}}+\tilde{\mathbf{D}} \dot{\tilde{\mathbf{q}}}+\tilde{\mathbf{K}} \tilde{\mathbf{q}}=\tilde{\mathbf{F}}_{\mathrm{ex}}+\tilde{\mathbf{R}}
$$

with the new DOF vector

$$
\tilde{\mathbf{q}}=\left[\begin{array}{c}
\mathbf{q}_{\mathrm{m}}(t) \\
\boldsymbol{\eta}(t)
\end{array}\right]
$$

now containing the modal DOF $\boldsymbol{\eta}(t)$ which replaced the original information $\mathbf{q}_{\mathrm{s}}(t)$ of the slave nodes.

\section{NEWMARK PREDICTOR CORRECTOR SCHEME}

When simulating periodic responses for linear or nonlinear systems, it has to be determined whether the computation should take place in frequency or time domain. In the proposed case, the time domain was chosen since it is known beforehand that impacts will occur - which are difficult to model and require high computational effort if represented by superposition of harmonics. After comparing several different time stepping schemes, the Newmark method was selected because of its high computational stability. Thus, forced response calculations can be performed with rather large time step sizes which results in decreased computation times. If combining the regular Newmark method

$$
\begin{aligned}
& \dot{\mathbf{q}}_{\mathrm{n}+1}=\dot{\mathbf{q}}_{\mathrm{n}}+\Delta t\left[\gamma \ddot{\mathbf{q}}_{\mathrm{n}+1}+(1-\gamma) \ddot{\mathbf{q}}_{\mathrm{n}}\right] \\
& \mathbf{q}_{\mathrm{n}+1}=\mathbf{q}_{\mathrm{n}}+\Delta t \dot{\mathbf{q}}_{\mathrm{n}}+\frac{\Delta t^{2}}{2}\left[2 \beta \ddot{\mathbf{q}}_{\mathrm{n}+1}+(1-2 \beta) \ddot{\mathbf{q}}_{\mathrm{n}}\right]
\end{aligned}
$$

with the differential equation of motion Eqn. (8), the resulting equation

$$
\tilde{\mathbf{q}}_{\mathrm{n}+1}=\tilde{\mathbf{K}}_{\mathrm{eff}}^{-1} \tilde{\mathbf{R}}+f\left(\tilde{\mathbf{F}}_{\mathrm{ex}}, \tilde{\mathbf{q}}_{\mathrm{n}}, \dot{\tilde{\mathbf{q}}}_{\mathrm{n}}, \ddot{\tilde{\mathbf{q}}}_{\mathrm{n}}\right)
$$

with

$$
\tilde{\mathbf{K}}_{\text {eff }}=\frac{1}{\beta \Delta t^{2}} \tilde{\mathbf{M}}+\frac{\gamma}{\beta \Delta t} \tilde{\mathbf{D}}+\tilde{\mathbf{K}}
$$

can be used do calculate the deflection at the end of the time step $\tilde{\mathbf{q}}_{\mathrm{n}+1}$ in the linear case, i.e. for $\tilde{\mathbf{R}}=\mathbf{0}$, since all necessary parameters are known. In the proposed method, this step - which is defined as the predictor step - is performed at the beginning of every time step. The vector $\tilde{\mathbf{q}}_{\mathrm{n}+1}$ is then employed to check if a contact is closed. If that is not the case, $\tilde{\mathbf{q}}_{\mathrm{n}+1}$ represents the true values at the end of the step. However, if contact is determined, the contact force vector $\tilde{\mathbf{R}}$ must be determined and a corrector step must be performed with a modified Newmark scheme of kind

$$
\begin{aligned}
& \dot{\mathbf{q}}_{\mathrm{n}+1}=\dot{\mathbf{q}}_{\mathrm{n}}+\Delta t\left[\gamma \ddot{\mathbf{q}}_{\mathrm{n}+1}+(1-\gamma) \ddot{\mathbf{q}}_{\mathrm{n}}\right]+\Delta t \ddot{\mathbf{q}}_{\mathrm{c}} \\
& \mathbf{q}_{\mathrm{n}+1}=\mathbf{q}_{\mathrm{n}}+\Delta t \dot{\mathbf{q}}_{\mathrm{n}}+\frac{\Delta t^{2}}{2}\left[2 \beta \ddot{\mathbf{q}}_{\mathrm{n}+1}+(1-2 \beta) \ddot{\mathbf{q}}_{\mathrm{n}}\right]+\frac{\Delta t^{2}}{2} \ddot{\mathbf{q}}_{\mathrm{c}}
\end{aligned}
$$

and a new differential equation of motion

$$
\mathbf{M}\left[\ddot{\tilde{\mathbf{q}}}_{\mathrm{n}+1}+\ddot{\tilde{\mathbf{q}}}_{\mathrm{c}}\right]+\mathbf{D} \dot{\tilde{\mathbf{q}}}_{\mathrm{n}+1}+\mathbf{K} \tilde{\mathbf{q}}_{\mathrm{n}+1}=\tilde{\mathbf{F}}_{\mathrm{ex}}+\tilde{\mathbf{R}} .
$$


The introduction of $\ddot{\tilde{q}}_{\mathrm{C}}$, which can be interpreted as a contact acceleration, is necessary to not introduce energy into the system. When performing the corrector step, the vector $\ddot{\tilde{q}}_{\mathrm{n}+1}$ must be adopted from the predictor step. Thus, the corrector step equation can be stated as

$$
\tilde{\mathbf{q}}_{\mathrm{n}+1}=\tilde{\mathbf{K}}_{\mathrm{eff}, \mathrm{c}}^{-1} \tilde{\mathbf{R}}+f\left(\tilde{\mathbf{F}}_{\mathrm{ex}}, \tilde{\mathbf{q}}_{\mathrm{n}}, \dot{\tilde{\mathbf{q}}}_{\mathrm{n}}, \ddot{\tilde{\mathbf{q}}}_{\mathrm{n}}, \ddot{\tilde{\mathbf{q}}}_{\mathrm{n}+1}\right)
$$

with

$$
\tilde{\mathbf{K}}_{\text {eff }, \mathrm{c}}=\frac{2}{\Delta t^{2}} \tilde{\mathbf{M}}+\frac{2}{\Delta t} \tilde{\mathbf{D}}+\tilde{\mathbf{K}}
$$

and serves as the true DOF vector at the end of the time step.

\section{THE CONTACT MODEL}

The proposed algorithm computes contact forces as Lagrangian multipliers by solving a LCP formulation. This formulation basically represents an enhanced combination of the methods proposed in [3] and [4] which the authors applied to rigid body structures. Since the systems simulated in these publications were neither FE based nor very stiff, factors like computation time and stability did not have to be payed attention to. That is why their formulations had to be rewritten, and the novel time stepping approach described in the previous chapter had to be included.

In order to enable the contact force computation by a LCP formulation, the contact force vector $\tilde{\mathbf{R}}$ has to be stated as follows:

$$
\begin{aligned}
\tilde{\mathbf{R}} & =\sum_{i} \mathbf{w}_{\mathrm{N}, i} \tilde{\Lambda}_{\mathrm{N}, i}+\mathbf{w}_{\mathrm{T}, i} \tilde{\Lambda}_{\mathrm{T}, i} \\
& =\mathbf{W}_{\mathrm{N}} \tilde{\Lambda}_{\mathrm{N}}+\mathbf{W}_{\mathrm{T}} \tilde{\Lambda}_{\mathrm{T}} .
\end{aligned}
$$

Herein, the vectors $\mathbf{w}_{\mathrm{N}, i}$ and $\mathbf{w}_{\mathrm{T}, i}$ stand for the normal and tangential contact force direction vectors which are being multiplied with the scalar force magnitudes $\tilde{\Lambda}_{\mathrm{N}, i}$ and $\tilde{\Lambda}_{\mathrm{T}, i}$. While the normal force direction vectors $\mathbf{w}_{\mathrm{N}, i}$ can simply be obtained by a geometric analysis of the cylindrical contact bodies involved, finding the tangential direction vectors $\mathbf{w}_{\mathrm{T}, i}$ must be done by projecting the velocity vector of the nodes in contact onto the friction plane defined by the normal vector $\mathbf{w}_{\mathrm{N}, i}$. Fig. 2 shows the tip of the beam depicted in Fig. 1 including a possible contact setup with the node touching the contact geometry marked black. The normal vector of the contact plane $\mathbf{w}_{\mathrm{N}}$ is facing towards the middle axis of the beam whereas $\mathbf{w}_{\mathrm{T}}$ is the projection of $\dot{\mathbf{q}}_{\mathrm{nc}}$ onto the grey friction plane. Obviously, this method is only applicable for $\left|\dot{\mathrm{q}}_{\mathrm{nc}}\right| \neq 0$, that is for slip friction. In case of sticking, the acceleration $\ddot{\mathbf{q}}_{\mathrm{nc}}$ has to be used instead.

A brief description of the original LCP derivation and its modification will be given in the following. For a more detailed explanation see also [3], [5] and [6]. In a first step, the Coulomb friction as well as the normal and tangential Newton impact models have to be stated as

$$
\begin{aligned}
\tilde{\boldsymbol{\Lambda}}_{\mathrm{T}} & =\mu \tilde{\boldsymbol{\Lambda}}_{\mathrm{N}}, \\
\boldsymbol{\xi}_{\mathrm{N}} & =\mathbf{W}_{\mathrm{N}}^{T} \dot{\tilde{\mathbf{q}}}_{\mathrm{n}+1}+\epsilon_{\mathrm{N}} \mathbf{W}_{\mathrm{N}}^{T} \dot{\tilde{\mathbf{q}}}_{\mathrm{n}}, \\
\boldsymbol{\xi}_{\mathrm{T}} & =\mathbf{W}_{\mathrm{T}}^{T} \dot{\tilde{\mathbf{q}}}_{\mathrm{n}+1}+\epsilon_{\mathrm{T}} \mathbf{W}_{\mathrm{T}}^{T} \dot{\tilde{\mathbf{q}}}_{\mathrm{n}}
\end{aligned}
$$




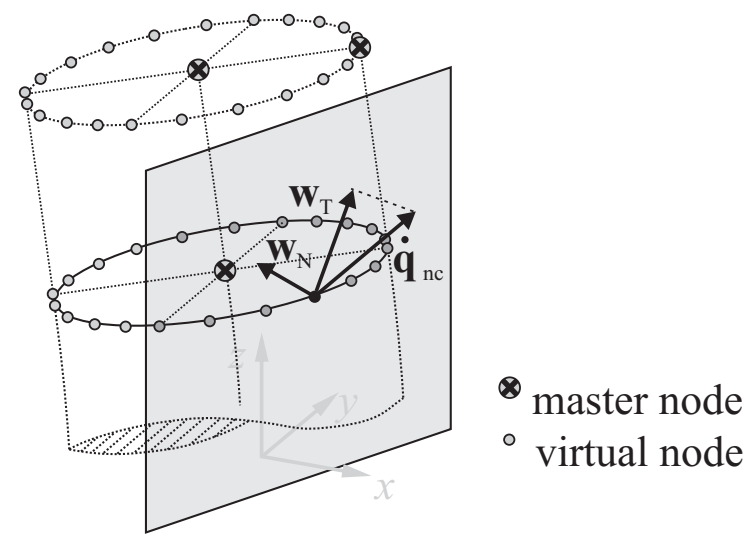

Figure 2. Beam tip showing a possible contact setup with the resulting force direction vectors and the three nodes necessary to describe the cylinder location

with normal and tangential force magnitudes $\Lambda_{\mathrm{N}}$ and $\Lambda_{\mathrm{T}}$, relative velocities in the contact point $\gamma_{\mathrm{N}}$ and $\gamma_{\mathrm{T}}$ and the coefficients of friction and restitution $\mu$ and $\epsilon$. The parameters $\boldsymbol{\xi}_{\mathrm{i}}$ have to be equal to zero for the Newton impact definition to be fulfilled. This formulation was stated by the authors of [3] - especially designed for the LCP formulation. Since the sign of the tangential force magnitude is not known yet, additional definitions have to be stated:

$$
\begin{aligned}
\tilde{\boldsymbol{\Lambda}}_{\mathrm{R}} & =\mu \tilde{\boldsymbol{\Lambda}}_{\mathrm{N}}+\tilde{\boldsymbol{\Lambda}}_{\mathrm{T}}, \\
\tilde{\boldsymbol{\Lambda}}_{\mathrm{L}} & =\mu \tilde{\boldsymbol{\Lambda}}_{\mathrm{N}}-\tilde{\boldsymbol{\Lambda}}_{\mathrm{T}}, \\
\boldsymbol{\xi}_{\mathrm{T}} & =\boldsymbol{\xi}_{\mathrm{R}}-\boldsymbol{\xi}_{\mathrm{L}} .
\end{aligned}
$$

Thus, the contact force vector $\tilde{\mathbf{R}}$ can be rewritten as

$$
\tilde{\mathbf{R}}=\left(\mathbf{W}_{\mathrm{N}}-\mu \mathbf{W}_{\mathrm{T}}\right) \tilde{\Lambda}_{\mathrm{N}}+\mathbf{W}_{\mathrm{T}} \tilde{\boldsymbol{\Lambda}}_{\mathrm{R}}
$$

If now using Eqn. (14) to Eqn. (16), an expression for $\dot{\tilde{\mathbf{q}}}_{\mathrm{n}+1}$ can be determined and employed into Eqn. (21) and Eqn. (22). The result is the linear equation

$$
\begin{aligned}
& {\left[\begin{array}{c}
\boldsymbol{\xi}_{\mathrm{N}} \\
\boldsymbol{\xi}_{\mathrm{R}} \\
\boldsymbol{\Lambda}_{\mathrm{L}}
\end{array}\right]=\left[\begin{array}{ccc}
\mathbf{W}_{\mathrm{N}}^{\mathrm{T}} \tilde{\mathbf{D}}_{\text {eff }}^{-1}\left(\mathbf{W}_{\mathrm{N}}-\mathbf{W}_{\mathrm{T}} \mu\right) & \mathbf{W}_{\mathrm{N}}^{\mathrm{T}} \tilde{\mathbf{D}}_{\text {eff }}^{-1} \mathbf{W}_{\mathrm{T}} & \mathbf{0} \\
\mathbf{W}_{\mathrm{T}}^{\mathrm{T}} \tilde{\mathbf{D}}_{\text {eff }}^{-1}\left(\mathbf{W}_{\mathrm{N}}-\mathbf{W}_{\mathrm{T}} \mu\right) & \mathbf{W}_{\mathrm{T}}^{\mathrm{T}} \tilde{\mathbf{D}}_{\text {eff }}^{-1} \mathbf{W}_{\mathrm{T}} & \mathbf{E} \\
2 \mu \mathbf{E} & -\mathbf{E} & \mathbf{0}
\end{array}\right]\left[\begin{array}{c}
\boldsymbol{\Lambda}_{\mathrm{N}} \\
\boldsymbol{\Lambda}_{\mathrm{R}} \\
\boldsymbol{\xi}_{\mathrm{L}}
\end{array}\right]} \\
& +\left[\begin{array}{c}
\mathbf{W}_{\mathrm{N}}^{\mathrm{T}} \tilde{\mathbf{D}}_{\text {eff }}^{-1} f\left(\tilde{\mathbf{F}}_{\mathrm{ex}}, \tilde{\mathbf{q}}_{\mathrm{n}}, \dot{\tilde{\mathbf{q}}}_{\mathrm{n}}, \ddot{\tilde{\mathbf{q}}}_{\mathrm{n}}, \ddot{\tilde{\mathbf{q}}}_{\mathrm{n}+1}\right)+\epsilon_{\mathrm{N}} \mathbf{W}_{\mathrm{N}}^{T} \dot{\tilde{\mathbf{q}}}_{\mathrm{n}} \\
\mathbf{W}_{\mathrm{T}}^{\mathrm{T}} \tilde{\mathbf{D}}_{\text {eff }}^{-1} f\left(\tilde{\mathbf{F}}_{\mathrm{ex}}, \tilde{\mathbf{q}}_{\mathrm{n}}, \dot{\tilde{\mathbf{q}}}_{\mathrm{n}}, \ddot{\tilde{\mathbf{q}}}_{\mathrm{n}}, \ddot{\tilde{\mathbf{q}}}_{\mathrm{n}+1}\right)+\epsilon_{\mathrm{T}} \mathbf{W}_{\mathrm{T}}^{T} \tilde{\tilde{\mathbf{q}}}_{\mathrm{n}} \\
0
\end{array}\right]
\end{aligned}
$$

with

$$
\tilde{\mathbf{D}}_{\text {eff }}=\frac{1}{\Delta t} \tilde{\mathbf{M}}+\tilde{\mathbf{D}}+\frac{\Delta t}{2} \tilde{\mathbf{K}}
$$

of kind $\mathbf{y}=\mathbf{A x}+\mathbf{b}$ with the complementary vectors $\mathbf{y}$ and $\mathbf{x}$, which can be solved utilizing algorithms like the one proposed by Lemke [7, 8]. Thus, the contact force vector can be determined according to Eqn. (26), and the time step solution may be obtained by Eqn. (17). 


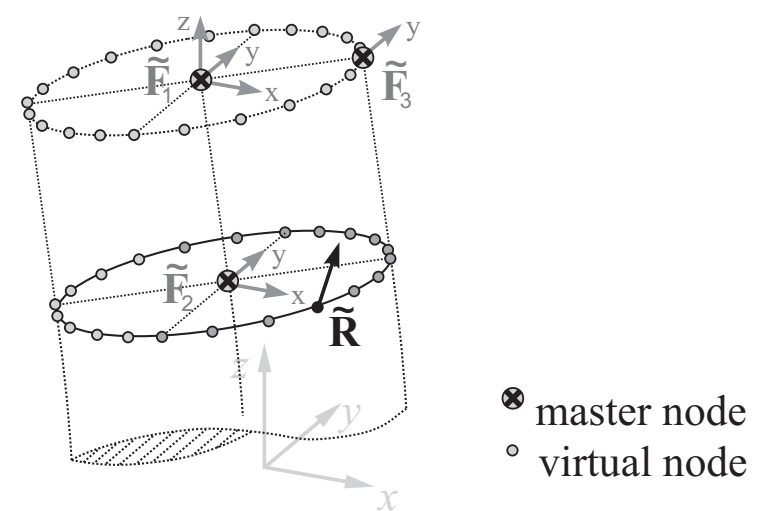

Figure 3. Distribution of the contact force on the virtual contact node among the master nodes

\section{CONTACT GEOMETRY DISCRETIZATION}

As mentioned before, the reduced order equation of motion still contains physical information about the nodes necessary to determine wether the beam is in contact with the bushing or wall. The number of these physical DOF should be kept at a minimum in order to decrease the size of the reduced structural matrices and thus, the computation time. That is why it makes sense to determine beforehand what kind and magnitude of deformation can be expected and which assumptions can be made to describe the locations and profiles of the contact surfaces involved with sufficient accuracy.

In the proposed case the component to be analyzed is a $300 \mathrm{~mm}$ steel alloy beam with a relatively small diameter of $9 \mathrm{~mm}$ (Fig. 1 is not true to scale). Since the rigid wall is located in a way that only the tip of the beam (last $20 \mathrm{~mm}$ of its length) can get in contact with it, it is valid to assume the deformations of the tip - radial as well as axial - to be negligible, i.e. the contact geometry to be a perfect cylinder. Position and orientation of this cylindrical geometry can be determined at every time step knowing the location of three of its nodes, which were chosen to be the ones at the top and bottom of its middle axis as well as an arbitrary one on its top edge (see Fig. 2). The proposed algorithm interpolates a user-defined number of virtual nodes along the two edges and checks these node locations for closed contacts. Note that the virtual node coordinates do not exist within the reduced DOF vector $\tilde{\mathbf{q}}$, which is why the force direction vectors $\mathbf{w}_{\mathrm{N}}$ and $\mathbf{w}_{\mathrm{T}}$ cannot be stated directly. That is why, after determining the contact force directions according to the previous chapter, these direction vectors must be applied to the according middle nodes in order to be able to build up the LCP. After determining the contact force $\tilde{\mathbf{R}}$, this force vector should not immediately be applied to Eqn. (17), but distributed first among the physically available DOF of the three master nodes shown in Fig. 2 and Fig. 3 adhering static equivalence of the contact force effect onto the cylinder:

$$
\begin{gathered}
\sum_{i} \tilde{\mathbf{F}}_{i}=\tilde{\mathbf{R}}, \\
\sum_{i} \mathbf{r}_{i} \times \tilde{\mathbf{F}}_{i}=\mathbf{r}_{\mathrm{c}} \times \tilde{\mathbf{R}} .
\end{gathered}
$$

If the original FE model only consisted of translational DOF, as is the case for the proposed beam model, nine DOF are available when considering three master nodes. However, only 
six force components are necessary in order to fulfill the static requirements stated above, which is why Fig. 3 only shows six contact force components of the nodal contact forces $\mathbf{F}_{i}$. The three remaining components were set to zero. The resulting contact force vector which applicable to Eqn. (17) can finally be stated as

$$
\tilde{\mathbf{R}}_{\mathrm{fin}}=\sum_{i} \tilde{\mathbf{F}}_{i}
$$

\section{DETECTION OF PERIODICITY}

The previously described methods alone are sufficient for the simulation. However, the simulation should stop automatically as soon as the system reached the steady state according to its harmonic excitation. Thus, periodic responses must be detected. That is why, starting with the second excitation period, the system's deflection and velocity vectors $\mathbf{q}_{1}$ and $\dot{\mathbf{q}}_{1}$ at the beginning of every period are being compared to the previous ones, and their relative compliance $c$ is being calculated. It is obvious that, in case of a periodic response over a single excitation period, the relative compliance approaches $c=100 \%$ (case A). If, however, a subharmonic response over several excitation periods appears, the compliance values will repeat (case B). Thus, subharmonic responses can be detected by locating these repeating compliance values. Examples of these two different cases are shown in Fig. 4.

After detecting the steady state response for a certain excitation frequency, the proposed algorithm locates the maximal response amplitude of certain predefined response nodes. These are, in case of the proposed structure, all nodes along the beam's middle axis. By plotting these maximal response amplitudes over the excitation frequency for varying clearances, response changes can be monitored and the clearance optimized.

A)

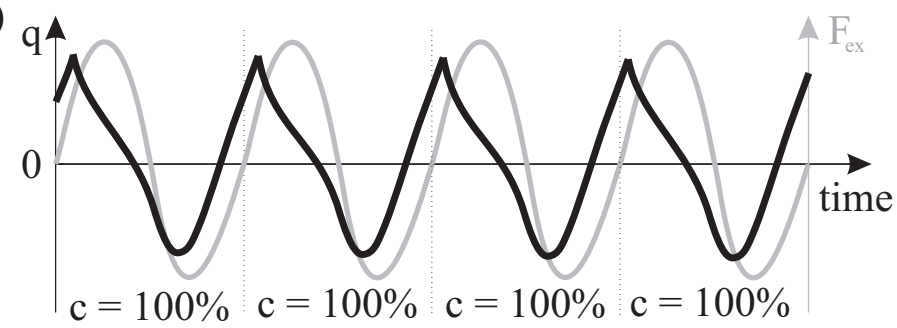

B)

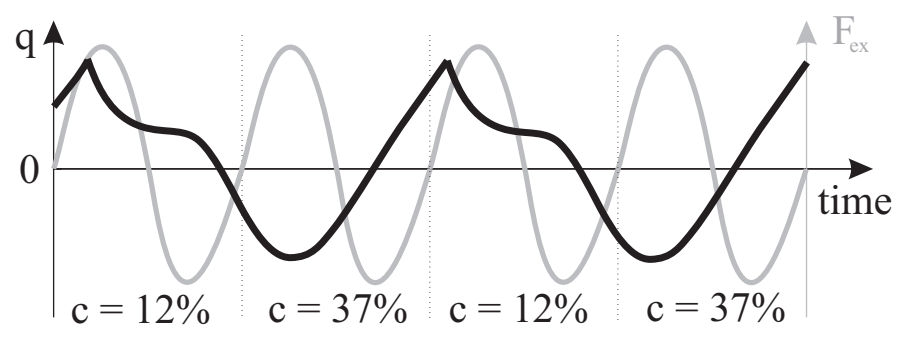

Figure 4. Examples for periodicity detection over A) one and B) two excitation periods 


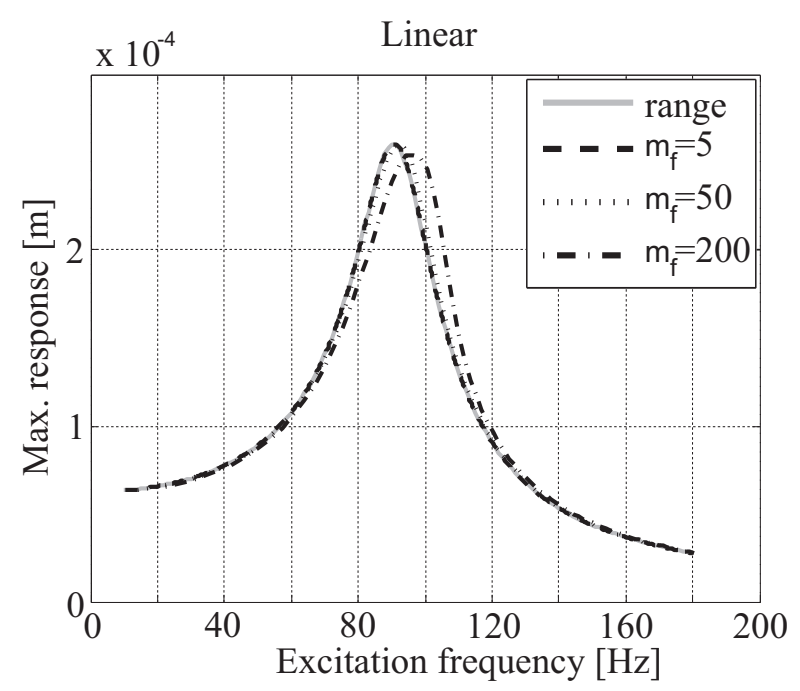

Figure 5. Periodic response behavior of the linear system

\section{SIMULATION RESULTS}

In the following chapter results are presented for the cylindrical beam with a rigid contact wall (Fig. 1B). The analysis leading to these results focussed on the first eigenfrequency of the beam located at approximately $90 \mathrm{~Hz}$. Since waiting for the steady state response to occur can, especially for metal structures with low damping coefficients, be fairly time consuming, additional frequency sweep simulations were performed. Herein, the same excitation frequency range was covered by excitations according to

$$
\mathbf{F}_{\mathrm{ex}}(t)=\hat{\mathbf{F}} \sin \left(2 \pi\left(f_{0}+\frac{1}{2} m_{\mathrm{f}} t\right) t\right)
$$

with frequency gain factors of $m_{\mathrm{f}}=5 \frac{\mathrm{Hz}}{\mathrm{s}}, m_{\mathrm{f}}=50 \frac{\mathrm{Hz}}{\mathrm{s}}$ and $m_{\mathrm{f}}=200 \frac{\mathrm{Hz}}{\mathrm{s}}$. Both harmonic and transient excitation forces were applied to the structure in a $45^{\circ}$ angle at note $n_{\text {exc }}$ (see Fig. 1B). Fig. 5 shows the response behavior of the linear system. The label "range" indicates the true steady state response whereas the three other curves represent the different sweep responses. As to be expected, the deviation of the swept amplitude frequency behavior from the steady state one increases for increasing frequency gain factors $m_{\mathrm{f}}$. Although the curves seem to converge for small values of $m_{\mathrm{f}}$, it shall be noted here that this convergence strongly depends on the structural damping.

In Fig. 6A and Fig. 6B, the same frequency range was simulated for the nonlinear system with the distances between beam and wall of $x_{\text {clear }}=2.4 \cdot 10^{-4} \mathrm{~m}$ and $x_{\text {clear }}=2.1 \cdot 10^{-4} \mathrm{~m}$. While the nonlinear behavior becomes increasingly distinctive as the clearance decreases, the frequency value showing the maximal amplitude does not noticeably shift. If looking at the actual movement of the beam, the reason for the absence of a frequency shift becomes obvious as the wall changes the orientation of the beam movement rather than blocking it, i.e. the beam's swinging orientation adjusts to the wall's contact surface. 

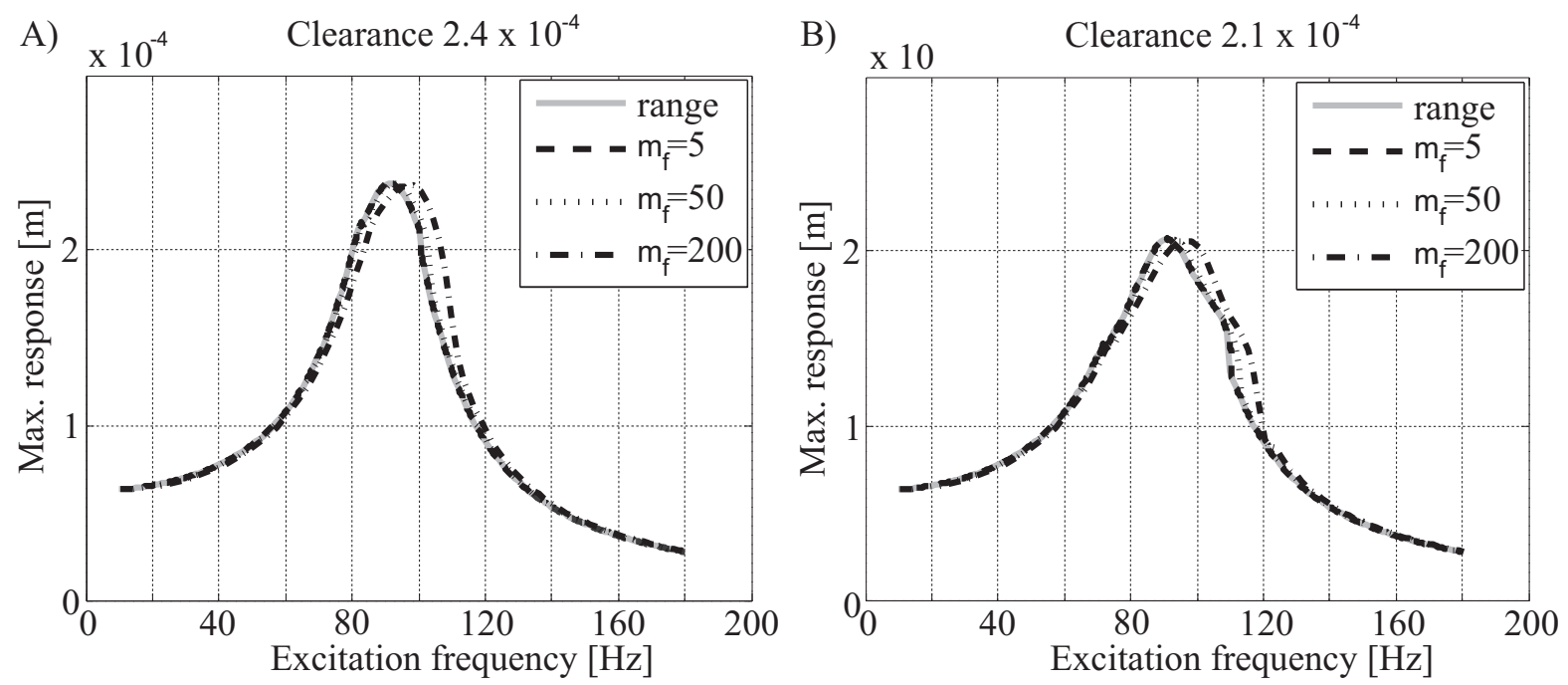

Figure 6. Periodic response behavior of nonlinear systems

\section{CONCLUSIONS AND PROSPECTS}

The proposed simulation method is capable of efficiently computing the forced response behavior of nonlinear structures. While commercial FE and multibody system software tools often neglect or significantly simplify the contact dynamics or structural properties, the proposed algorithms use the strength of FE models - which is the accurate description of the structure - and combine it with a dynamic simulation method which considers separation, stick and slip friction as well as impacts occurring after separation intervals. The functionality has been demonstrated on a simple structure of a cylindrical beam with a rigid contact wall in order to give a detailed description of the implemented algorithms.

Future work will focus on the experimental validation of the simulation model on both simple structures and true machinery components. Possible improvements like the utilization of shooting approaches (see e.g. [9] and [10]) for a further efficiency increase through computation time minimization will be discussed before applying the code to full machinery systems.

\section{Acknowledgements}

The work described in this paper has been performed within the national research project Kontakte mit Spiel funded by the Arbeitsgemeinschaft industrieller Forschungsvereinigungen (AiF) and the Forschungsvereinigung Verbrennungskraftmaschinen e.V.(FVV).

\section{References}

[1] Craig, R. R., and Bampton, M. C., 1968. "Coupling of Substructures for Dynamic Analysis". AIAA Journal, 6(7), pp. 1313-1319.

[2] Cook, R., Malkus, D., Plesha, M., and Witt, R., 2001. Concepts and Applications of Finite Element Analysis, 4 ed. John Wiley \& Sons. 
[3] Glocker, C., and Studer, C., 2003. "The Woodpecker Toy". Proceedings of the IMES Center for Mechanics, ETH Zurich.

[4] Tatzko, S., Grönefeld, P., and Wangenheim, M., 2009. “Application of LCP Contact Formulation on Mechanical Systems With Nonrigid Coupled Unilateral Contact Points Illustrated With a Simple Model of a Windscreen Wiper". Proceedings of The International Mechanical Engineering Congress and Exposition IMECE(IMECE2009-10292), pp. 435-442.

[5] Pfeiffer, F., and Glocker, C., 2000. Multibody dynamics with unilateral contacts, 1 ed. John Wiley \& Sons.

[6] Schurzig, D., Tatzko, S., Panning-von Scheidt, L., and Wallaschek, J., 2012. Modeling Contact Dynamics of Vanes with Adjustable Upstream Flow Angles. Paper GT201268185, Proc. of ASME Turbo Expo 2012: GT2012, June 11-15, Copenhagen, Denmark.

[7] Lemke, C., 1965. "Bimatrix Equilibrium Points and Mathematical Programming". Management Science 11(7), pp. 681-689.

[8] Tomlin, J., 1978. "Robust implementation of Lemke's method for the linear complementarity problem". Mathematical Programming Study 7(7), pp. 55-60.

[9] Patel, B., Ibrahim, S., and Nath, Y., 2009. "Periodic response of nonlinear dynamical system with large number of degrees of freedom". Sadhana 34(6), pp. 1033-1037.

[10] Ibrahim, S., Patel, B., and Nath, Y., 2009. "Modified shooting approach to the non-linear periodic forced response of isotropic/composite curved beams". International Journal of Non-Linear Mechanics 44, pp. 1073-1084. 